\title{
Triple -Diffusive Convection in a Magnetized Ferrofluid With MFD Viscosity: A Nonlinear Stability Analysis
}

\author{
Dr. Suresh Chand \\ Department of Mathematics, SCVB Govt. College Palampur, HP-176061 India
}

\begin{abstract}
A nonlinear stability analysis is performed for a triple-diffusive convection in a magnetized ferrofluid with magnetic field -dependent viscosity (MFD) for stress- free boundaries. The major mathematical emphasis is on how to control the non-linear terms caused by magnetic body force and inertia forces. A suitable generalized energy functional is introduced to perform the nonlinear energy stability analysis. It is found that nonlinear critical stability magnetic thermal Rayleigh number does not coincide with that of linear instability, and thus indicate that the subcritical instabilities are possible. However, it is noted that in case of non-ferrofluid global nonlinear stability Rayleigh number is exactly same as that of linear instability. For lower values of magnetic parameters, this coincidence is immediately lost. The effects of magnetic parameter $M_{3}$, solute gradients $S_{1} \& S_{2}$ and MFD viscosity parameter $\delta$, on the subcritical instability region have also been analyzed. The solutes gradients $S_{1} \& S_{2}$ have stabilizing effect, because both $N_{c e}, N_{c \ell}$ increases as solute gradients increases. It has also been observed that in the presence of MFD viscosity $(\delta)$, both $N_{c e}, N_{c \ell}$ decrease for lower values of $M_{3}$ and increase for higher values of $M_{3}$.
\end{abstract}

Keywords: nonlinear stability, magnetized ferrofluid, triple- diffusive convection, MFD viscosity , magnetization.

\section{Introduction}

Magnetic fluids or ferrofluids are colloidal suspension of fine ferromagnetic mono domain nano particles in non-conducting liquids. The ferromagnetic nanoparticles are coated with a surfactant to prevent their agglomeration. Rosensweig [1985] in his monograph and review article provides a detailed introduction to this subject. Chandrashekher [1981] has given a detailed account of thermal convection problems of Newtonian fluids. The theory of convective instability of ferrofluid begins with Finalyson [1970] and is interestingly continued by Lalas and Carmi [1971], Shliomis [1974], Stile and Kagan [1990], Venkatasubramanian and Kaloni [1994] .In the absence of an applied magnetic field, the particles in the colloidal suspensions are randomly oriented and thus the fluid has no net magnetization. When exposed to a magnetic field, Brownian rotational motions prevent complete alignment of the dipoles with the applied field. As a result when the applied field has a changing direction or magnitude, the magnetization is unable to track the field closely and becomes non-equilibrated. This non-equilibrium state of magnetization leads to the state of asymmetric stress. Rayleigh Bénard convection in a ferromagnetic fluid layer with internal angular momentum permeated by uniform, vertical magnetic field with free-free, isothermal, spin-vanishing, magnetic boundaries has been considered by Abraham [2002]. She observed that the micropolar ferromagnetic fluid layer heated from below is more stable as compared with the classical Newtonian ferromagnetic fluid. More recently, Suresh [2012] has studied the convection problems in a ferrofluid with internal angular momentum in a porous and non-porous medium.

In the standard Bénard problem, the instability is driven by a density difference caused by a temperature difference between the upper and lower planes bounding the fluid. If the fluid additionally has salt dissolved in it, then there are potentially two destabilizing sources for the density difference, the temperature field and salt field. The solution behavior in the double-diffusive convection problem is more interesting than that of the single component situation in so much as new instability phenomena may occur which is not present in the classical Bénard problem. When temperature and two or more component agencies, or three different salts, are present then the physical and mathematical situation becomes increasingly richer. Very interesting results in triply diffusive convection have been obtained by Pearlstein et al., [1989]. The results of Pearlstein et al., are remarkable. They demonstrate that for triple diffusive convection linear instability can occur in discrete sections of the Rayleigh number domain with the fluid being linearly stable in a region in between the linear instability ones. This is because for certain parameters the neutral curve has a finite isolated oscillatory instability curve lying below the usual unbounded stationary convection one. Straughan and Walker [1987] derive the equations for non-Boussinesq convection in a multi- component fluid and investigate the situation analogous to that of Pearlstein et al., but allowing for a density non linear in the temperature field. Lopez et al., [1990] derive the equivalent problem with fixed boundary conditions and show that the effect of the boundary conditions breaks the perfect symmetry. In reality the density of a fluid is never a linear function of temperature, and so the work of Straughan and Walker applies to the general situation where the equation of state is one of 
the density quadratic in temperature. This is important, since they find that departure from the linear Boussinesq equation of state changes the perfect symmetry of the heart shaped neutral curve of Pearlstein et al.,. A comprehensive review of the literature concerning convection in porous medium may be found in the book by Nield and Bejan [1998].

There are in general two methods in a stability analysis, the linearlized instability method and energy method. The linear stability method provides sufficient condition for instability, whereas the energy method provides sufficient condition of stability of a basic flow. It is also noticed that the linearized theory alone cannot decide whether a particular flow is stable or not, for this it requires it's response to all physically accepted disturbances. The energy methods on other hand guarantee the exponential decay of arbitrary disturbances at all times and thus can be fully conservative in determining the stable -unstable bounds. The energy method is one of the oldest methods for nonlinear stability and can be traced back to the work of Reynolds (1895) and Orr (1907). The revival of energy method has been acknowledged after the work of Serrin (1959) and Joseph (1965, 1966). Energy methods of nonlinear stability theory are based on the study of time evolution of energy of the perturbation to the basic flow, and leads to variational problem for a critical dimensionless number, below which energy decays to zero. The detailed discussion of literature pertinent to the energy method can be found in Straughan (2001). By introducing the coupling parameters in the energy method and by selecting them optimally, it has been possible to sharpen the stability bound in many physical problems as discussed by Straughan (2004). Nonlinear energy stability analysis for thermal convection with temperature - dependent viscosity in the fluid has been considered by Hill and Carr (2010). A problem of thermal convection in a fluidsaturated porous layer using a global nonlinear stability analysis with a thermal non-equilibrium model has been study by Straughan (2006). He established that the global nonlinear stability boundary obtained using LTNE theory is exactly the same as the linear instability theory by Banu and Rees (2002).Recently a nonlinear stability analysis of magnetized ferrofluid and the same problem in the porous media have been studied by Sunil and Mahajan $(2008,2009)$.

In this paper, I have studied the nonlinear stability analysis of triple- diffusive convection in a magnetized ferrofluid with MFD viscosity by using generalized energy method. This problem, to the best of my knowledge has not been analyzed yet. It is found that when buoyancy magnetization is absent i.e. in case of nonferrofluid, there is a coincidence between the nonlinear and linear stability results. For a convection problem in magnetized ferrofluid, the linear critical magnetic thermal Rayleigh number is found higher in values than the nonlinear critical magnetic thermal Rayleigh number, which shows the possibility of the existence of subcritical instability. Finally, the comparison of the results obtained, respectively, by the linear stability analysis and energy method has been discussed in detail.

\section{Mathematical formulation of the problem}

Here we consider an infinite, horizontal layer of thickness $d$ of an electrically non-conducting incompressible thin -magnetized ferrofluid heated and salted from below having variable viscosity $\mu_{1}=\mu$ $(1+\delta . B)$. The temperature $\mathrm{T}$ and solute concentrations $\mathrm{C}^{1}$ and $\mathrm{C}^{2}$ at the bottom and top surfaces $\mathrm{z}= \pm \frac{1}{2} d$ are $\mathrm{T}_{0}$ and $\mathrm{T}_{1} ; \mathrm{C}_{0}{ }^{1}$ and $\mathrm{C}_{1}{ }^{1}$; and $\mathrm{C}_{0}{ }^{2}$ and $\mathrm{C}_{1}{ }^{2}$ respectively, and a uniform temperature gradient $\beta\left(=\left|\frac{d T}{d Z}\right|\right)$ and uniform solute gradients are $\beta^{\prime}\left(=\left|\frac{d C^{1}}{d Z}\right|\right)$ and $\beta^{\prime \prime}\left(=\left|\frac{d C^{2}}{d Z}\right|\right)$ are maintained. Both the boundaries are taken to be free and perfect conductors of heat. The gravity field $\mathbf{g}=(0,0,-\mathrm{g})$ and uniform vertical magnetic field intensity $\mathbf{H}=\left(0,0, \mathrm{H}_{0}\right)$ pervade the system. The mathematical equations to discuss the nonlinear stability analysis in triple diffusive convection, for the above model are as follows ( Finlayson[1970]) : The continuity equation is

$\nabla \cdot \boldsymbol{q}=0$

The momentum equation is

$\rho_{0}\left[\frac{\partial}{\partial t}+(\mathbf{q} \cdot \nabla)\right] \mathbf{q}=-\nabla \mathrm{p}+\mu_{1} \nabla^{2} \mathrm{q}+\rho_{0}\left[1-\alpha\left(\mathrm{T}-\mathrm{T}_{\mathrm{a}}\right)+\alpha^{\prime}\left(\mathrm{C}^{1}-\mathrm{C}_{\mathrm{a}}^{1}\right)+\alpha^{\prime \prime}\left(\mathrm{C}^{2}-\mathrm{C}_{\mathrm{a}}^{2}\right)\right]+\mu_{0}(\mathbf{M} \cdot \nabla) \mathbf{H}$

The temperature and solute concentration equations are

$$
\begin{aligned}
& {\left[\frac{\partial}{\partial t}+(\mathbf{q} \cdot \nabla)\right] \mathbf{T}=\mathrm{K}_{1} \nabla^{2} \mathrm{~T}} \\
& {\left[\frac{\partial}{\partial t}+(\mathbf{q} \cdot \nabla)\right] \mathrm{C}^{1}=\mathrm{K}^{\prime}{ }_{1} \nabla^{2} \mathrm{C}^{1}} \\
& {\left[\frac{\partial}{\partial t}+(\mathbf{q} \cdot \nabla)\right] \mathrm{C}^{2}=\mathrm{K}_{1}{ }^{\prime}{ }^{\prime \prime} \nabla^{2} \mathrm{C}^{2}}
\end{aligned}
$$

Maxwell's equation, simplified for a non-conducting fluid with no displacement currents, become 
$\nabla . \mathbf{B}=0, \quad \nabla \times \mathbf{H}=0 \quad \mathbf{B}=\mu_{0}(\mathbf{H}+\mathbf{M})$.

We assume that the magnetization is aligned with the magnetic field, but allow a dependence on the magnitude of the magnetic field, temperature and salinity, so that

$\mathbf{M}=\frac{\boldsymbol{H}}{H} \mathrm{M}\left(\mathrm{H}, \mathrm{T}, \mathrm{C}^{1}, \mathrm{C}^{2}\right)$.

The magnetic equation of state is linearized about the magnetic field, $\mathrm{H}_{0}$, an average temperature, $T_{a}$, and average concentrations, $C_{a}{ }^{1}$ and $C_{a}{ }^{2}$ to become

$\mathrm{M}=\mathrm{M}_{0}+\chi\left(\mathrm{H}-\mathrm{H}_{0}\right)-\mathrm{K}_{2}\left(\mathrm{~T}-\mathrm{T}_{\mathrm{a}}\right)+\mathrm{K}_{3}\left(\mathrm{C}^{1}-\mathrm{C}_{\mathrm{a}}{ }^{1}\right)+\mathrm{K}_{4}\left(\mathrm{C}^{2}-\mathrm{C}_{\mathrm{a}}{ }^{2}\right)$.

where magnetic susceptibility, pyromagnetic coefficient and salinity magnetic coefficients are defined by

$\chi \equiv\left(\frac{\partial M}{\partial H}\right) \mathrm{H}_{0}, \mathrm{~T}_{\mathrm{a}} ; \quad \mathrm{K}_{2} \equiv-\left(\frac{\partial M}{\partial T}\right) \mathrm{H}_{0}, \mathrm{~T}_{\mathrm{a}} ; \mathrm{K}_{3} \equiv\left(\frac{\partial M}{\partial C^{1}}\right) \mathrm{H}_{0}, \mathrm{c}_{\mathrm{a}}{ }^{1}$ and $\mathrm{K}_{4} \equiv\left(\frac{\partial M}{\partial C^{2}}\right) \mathrm{H}_{0}, \mathrm{c}_{\mathrm{a}}{ }^{2}$ respectively.

Here $\mathrm{H}_{0}$ is the uniform magnetic field of the fluid layer when placed in an external magnetic field $\mathrm{H}=\mathrm{H}_{0}{ }^{\text {ext }} \hat{k}$, where $\hat{k}$ is a unit vector in the $\mathrm{z}$ direction, $\mathrm{H}=|\mathbf{H}|, \mathrm{M}=|\mathbf{M}|$ and $\mathrm{M}_{0}=\mathrm{M}\left(\mathrm{H}_{0}, \mathrm{~T}_{\mathrm{a}}, \mathrm{C}_{\mathrm{a}}{ }^{1}, \mathrm{C}_{\mathrm{a}}{ }^{2}\right)$

The basic state is assumed to be quiescent state and is given by

$\mathbf{q}=\mathbf{q}_{\mathrm{b}}=(0,0,0), \rho=\rho_{\mathrm{b}}(\mathrm{z}), \mathrm{p}=\mathrm{p}_{\mathrm{b}}(\mathrm{z}), \mathrm{T}=\mathrm{T}_{\mathrm{b}}(\mathrm{z})=-\beta z+\mathrm{T}_{\mathrm{a}}, \mathrm{C}^{1}=\mathrm{C}_{\mathrm{b}}^{1}(\mathrm{z})=-\beta^{\prime} z+C^{1}{ }_{\mathrm{a}}$

$\mathrm{C}^{2}=\mathrm{C}_{\mathrm{b}}^{2}(\mathrm{z})=-\beta^{\prime \prime} z+C_{\mathrm{a}}^{2}, \quad \beta=\left(\mathrm{T}_{0}-\mathrm{T}_{1}\right) / \mathrm{d}, \quad \beta^{\prime}=\left(\mathrm{C}_{1}{ }^{1}-\mathrm{C}_{0}{ }^{1}\right) / \mathrm{d}, \quad \beta^{\prime \prime}=\left(\mathrm{C}_{1}{ }^{2}-\mathrm{C}_{0}{ }^{2}\right) / \mathrm{d}$,

$\mathbf{H}_{\mathbf{b}}=\left[\mathrm{H}_{0}-\frac{K_{2} \beta \mathrm{z}}{1+\chi}+\frac{K_{3} \beta^{\prime} \mathrm{z}}{1+\chi}+\frac{K_{4} \beta^{\prime \prime} \mathrm{z}}{1+\chi}\right] \widehat{\boldsymbol{k}}, \mathbf{M}_{\mathbf{b}}=\left[\mathrm{M}_{0}+\frac{K_{2} \beta \mathrm{z}}{1+\chi}-\frac{K_{3} \beta^{\prime} \mathrm{z}}{1+\chi}-\frac{K_{4} \beta^{\prime \prime} \mathrm{z}}{1+\chi}\right] \widehat{\boldsymbol{k}}$ and $\mathrm{H}_{0}+\mathrm{M}_{\mathrm{O}}=\mathrm{H}_{0}{ }^{\mathrm{ext}}$,

where the subscript " $b$ " denotes the basic state.

We now examine the stability of the basic state, and assume that the perturbation quantities are small. We write

$\mathbf{q}=\mathbf{q}_{\mathbf{b}}+\mathbf{q}^{\prime}, \rho=\rho_{\mathrm{b}}+\rho^{\prime}, \mathbf{p}=\mathrm{p}_{\mathrm{b}}(\mathrm{z})+\mathrm{p}^{\prime}, \quad \mathrm{T}=\mathrm{T}_{\mathrm{b}}(\mathrm{z})+\theta, \mathrm{C}^{1}=\mathrm{C}_{\mathrm{b}}^{1}(\mathrm{z})+\gamma, \mathrm{C}^{2}=\mathrm{C}_{\mathrm{b}}^{2}(\mathrm{z})+\gamma^{\prime}$,

$\mathbf{H}=\mathbf{H}_{\mathrm{b}}(\mathrm{z})+\mathbf{H}^{\prime}$ and $\mathbf{M}=\mathbf{M}_{\mathrm{b}}(\mathrm{z})+\mathbf{M}^{\prime}$

where $\mathbf{q}^{\prime}=(\mathrm{u}, \mathrm{v}, \mathrm{w}), \rho^{\prime}, \mathrm{p}^{\prime}, \theta, \gamma, \gamma^{\prime}, \mathbf{H}^{\prime}, \mathbf{M}^{\prime}$ are perturbation in velocity $\mathrm{q}$, pressure $\mathrm{p}$, temperature $\mathrm{T}$, concentrations $\mathrm{C}^{1}$ and $\mathrm{C}^{2}$, magnetic field intensity $\mathbf{H}$, and magnetization $\mathbf{M}$, respectively. The change in density $\rho^{\prime}$, caused mainly by the perturbations $\theta, \gamma$, and $\gamma^{\prime}$ in temperature and concentrations, respectively, is given by

$\rho^{\prime}=-\rho_{0}\left(\alpha \theta-\alpha^{\prime} \gamma-\alpha^{\prime \prime} \gamma^{\prime}\right)$

The non-dimensionless equations for the perturbation are

$$
\begin{aligned}
& \frac{\partial \mathbf{q}}{\partial \mathrm{t}}=-\nabla \mathrm{p}+\left(1+\delta \mathrm{M}_{3}\right) \nabla^{2} \mathbf{q}+\sqrt{R}\left(1+\mathrm{M}_{1}-\mathrm{M}_{4}\right) \theta \widehat{\boldsymbol{k}}-\frac{\sqrt{S_{1}}}{L_{e}}\left(1+\mathrm{M}_{4}^{\prime}-\mathrm{M}_{1}^{\prime}\right) \gamma \widehat{\boldsymbol{k}}-\frac{\sqrt{S_{2}}}{L_{e}}\left(1+\mathrm{M}_{4}^{\prime \prime}-\mathbf{M}_{1}^{\prime \prime}\right) \gamma \widehat{\boldsymbol{k}} \\
& -\sqrt{R}\left(\mathrm{M}_{1}-\mathrm{M}_{4}\right) \phi_{1 \mathrm{Z}} \widehat{\boldsymbol{k}}+\frac{\sqrt{S_{1}}}{L_{e}}\left(\mathrm{M}_{4}^{\prime}-\mathrm{M}_{1}^{\prime}\right) \phi_{2 \mathrm{Z}} \widehat{\boldsymbol{k}}+\frac{\sqrt{S_{2}}}{L_{e}}\left(\mathrm{M}_{4}{ }^{\prime \prime}-\mathrm{M}_{1}{ }^{\prime \prime}\right) \phi_{3 \mathrm{Z}} \widehat{\boldsymbol{k}}-\mathrm{M}_{1} \theta \phi_{1 \mathrm{Z}}+\frac{\sqrt{M_{4}} \sqrt{M_{4}}}{\sqrt{L_{e}}}\left(\theta \nabla \phi_{2 \mathrm{Z}}\right. \\
& \left.+\gamma \phi_{1 \mathrm{Z}}\right)+\frac{\sqrt{M_{4}} \sqrt{M_{4}^{\prime \prime}}}{\sqrt{L_{e}}}\left(\theta \nabla \phi_{3 \mathrm{Z}}+\gamma^{\prime} \phi_{1 \mathrm{Z}}\right)+\frac{\sqrt{M^{\prime}} \sqrt{M_{4^{\prime \prime}}}}{\sqrt{L_{e}}}\left(\gamma \nabla \phi_{3 \mathrm{Z}}+\gamma^{\prime} \phi_{2 \mathrm{Z}}\right)+\left(\mathrm{M}_{3}-\frac{1}{1+\chi}\right)\left[\mathrm{M}_{1} \phi_{1 \mathrm{x}} \nabla \phi_{1 \mathrm{x}}-\frac{\sqrt{M_{4}} \sqrt{M_{4}}}{\sqrt{L_{e}}}\right. \\
& \left(\phi_{1 \mathrm{x}} \nabla \phi_{2 \mathrm{x}}+\phi_{2 \mathrm{x}} \nabla \phi_{1 \mathrm{x}}\right)-\frac{\sqrt{M_{4}} \sqrt{M_{4}^{\prime \prime}}}{\sqrt{L_{e}}}\left(\phi_{1 \mathrm{x}} \nabla \phi_{3 \mathrm{x}}+\phi_{3 \mathrm{x}} \nabla \phi_{1 \mathrm{x}}-\frac{\sqrt{M^{\prime}} \sqrt{M_{4}^{\prime \prime}}}{\sqrt{L_{e}}}\left(\phi_{2 \mathrm{x}} \nabla \phi_{3 \mathrm{x}}+\phi_{3 \mathrm{x}} \nabla \phi_{2 \mathrm{x}}\right)+\frac{M_{1}^{\prime}}{L_{e}} \phi_{2 \mathrm{x}} \nabla \phi_{2 \mathrm{x}}\right. \\
& \left.+\frac{M_{1}^{\prime \prime}}{L_{e}} \phi_{3 \mathrm{x}} \nabla \phi_{3 \mathrm{x}}\right]+\left(\mathrm{M}_{3}-\frac{1}{1+\chi}\right)\left[\mathrm{M}_{1} \phi_{1 \mathrm{y}} \nabla \phi_{1 \mathrm{y}}-\frac{\sqrt{M_{4}} \sqrt{M_{4}^{\prime \prime}}}{\sqrt{L_{e}}}\left(\phi_{1 \mathrm{y}} \nabla \phi_{2 \mathrm{y}}+\phi_{2 \mathrm{y}} \nabla \phi_{1 \mathrm{y}}-\frac{\sqrt{M_{4}} \sqrt{M_{4}{ }^{\prime \prime}}}{\sqrt{L_{e}}}\left(\phi_{1 \mathrm{y}} \nabla \phi_{3 \mathrm{y}}+\phi_{3 \mathrm{y}}\right.\right.\right. \\
& \left.\left.\nabla \phi_{1 \mathrm{y}}\right)-\frac{\sqrt{M^{\prime}} \sqrt{M_{4}^{\prime \prime}}}{\sqrt{L_{e}}}\left(\phi_{2 \mathrm{y}} \nabla \phi_{3 \mathrm{y}}+\phi_{3 \mathrm{y}} \nabla \phi_{2 \mathrm{y}}\right)+\frac{M_{1}{ }^{\prime}}{L_{e}} \phi_{2 \mathrm{y}} \nabla \phi_{2 \mathrm{y}}+\frac{M_{1}^{\prime \prime}}{L_{e}} \phi_{3 \mathrm{y}} \nabla \phi_{3 \mathrm{y}}\right]+\left(\frac{\chi}{1+\chi}\right)\left[\mathrm{M}_{1} \phi_{1 \mathrm{z}} \nabla \phi_{1 \mathrm{z}}\right. \\
& -\frac{\sqrt{M_{4}} \sqrt{M_{4}}}{\sqrt{L_{e}}}\left(\phi_{1 \mathrm{z}} \nabla \phi_{2 \mathrm{z}}+\phi_{2 \mathrm{z}} \nabla \phi_{1 \mathrm{z}}\right)-\frac{\sqrt{M_{4}} \sqrt{M_{4}^{\prime \prime}}}{\sqrt{L_{e}}}\left(\phi_{1 \mathrm{z}} \nabla \phi_{3 \mathrm{z}}+\phi_{3 \mathrm{z}} \nabla \phi_{1 \mathrm{z}}\right)-\frac{\sqrt{M_{4}^{4}} \sqrt{M_{4}^{\prime \prime}}}{\sqrt{L_{e}}}\left(\phi_{2 \mathrm{z}} \nabla \phi_{3 \mathrm{z}}+\phi_{3 \mathrm{z}} \nabla \phi_{2 \mathrm{z}}\right)
\end{aligned}
$$


$\left.-M_{\delta}{ }^{\prime} \phi_{2 y}-M_{\delta}{ }^{\prime \prime} \phi_{3 y}\right) \nabla^{2} \mathbf{q}+\delta\left(M_{\delta} \phi_{1 z}-M_{\delta}^{\prime} \phi_{2 z}-M_{\delta}{ }^{\prime \prime} \phi_{3 z}\right) \nabla^{2} \mathbf{q}-\delta M_{\delta} \theta \nabla^{2} \mathbf{q}+\delta M_{\delta}{ }^{\prime} \gamma \nabla^{2} \mathbf{q}+\delta M_{\delta}{ }^{\prime \prime} \gamma \nabla^{2} \mathbf{q}$

$\nabla \cdot \mathbf{q}=0$

$\frac{\partial \theta}{\partial \mathrm{t}}+\mathbf{q} \cdot \nabla \theta=\nabla^{2} \theta^{+} \sqrt{R} \mathrm{w}$

$\frac{\partial \gamma}{\partial \mathrm{t}}+\mathbf{q} \cdot \nabla \gamma=\frac{1}{L_{e}} \nabla^{2} \gamma+\sqrt{S_{1}} \mathrm{w}$

$\frac{\partial \gamma^{\prime}}{\partial \mathrm{t}}+\mathbf{q} \cdot \nabla \gamma^{\prime}=\frac{1}{L_{e}} \nabla^{2} \gamma^{\prime}+\sqrt{S_{2}} \mathrm{~W}$

$\mathrm{M}_{3} \nabla^{2} \phi_{1}-\left(\mathrm{M}_{3}-1\right) \phi_{1 \mathrm{zz}}=\theta_{\mathrm{z}}$

$\mathrm{M}_{3} \nabla^{2} \phi_{2}-\left(\mathrm{M}_{3}-1\right) \phi_{2 \mathrm{zz}}=\gamma_{\mathrm{z}}$

$\mathrm{M}_{3} \nabla^{2} \phi_{3}-\left(\mathrm{M}_{3}-1\right) \phi_{3 z z}=\gamma_{\mathrm{z}}^{\prime}$

Here, the following non dimension quantities and non dimensionless parameters are introduced:

$$
\begin{aligned}
& \gamma^{*}=\frac{\sqrt{S_{1}}}{\beta^{\prime} d} \gamma, \gamma^{\prime *}=\frac{\sqrt{S_{2}}}{\beta^{\prime \prime} d} \gamma^{\prime}, \quad \phi_{1}{ }^{*}=\frac{(1+\chi) \sqrt{R}}{K_{1} \beta d^{2}} \phi_{1}, \quad \phi_{2}{ }^{*}=\frac{(1+\chi) \sqrt{S_{1}}}{K_{2} \beta^{\prime} d^{2}} \phi_{2}, \quad \phi_{3}{ }^{*}=\frac{(1+\chi) \sqrt{S_{2}}}{K_{3} \beta^{\prime \prime} d^{2}} \phi_{3}, \\
& \delta^{*}=\mu_{0} \mathrm{H}_{0}(1+\chi) \delta, \quad \mathrm{R}=\frac{\mathrm{g} \alpha \beta \rho_{0} d^{4}}{\mu K_{1}}, \mathrm{~S}_{1}=\frac{\mathrm{g} \alpha^{\prime} \beta^{\prime} \rho_{0} d^{4}}{\mu K_{1}{ }^{\prime}}, \mathrm{S}_{2}=\frac{\mathrm{g} \alpha^{\prime \prime} \beta^{\prime \prime} \rho_{0} d^{4}}{\mu K_{1}{ }^{\prime}}, \mathrm{M}_{1}=\frac{\mu_{0} K_{1}^{2} \beta}{(1+\chi) \alpha \rho_{0} \mathrm{~g}}, \\
& \mathrm{M}_{1}^{\prime}=\frac{\mu_{0} K_{2}^{2} \beta^{\prime}}{(1+\chi) \alpha^{\prime} \rho_{0} \mathrm{~g}}, \quad \mathrm{M}_{1}^{\prime \prime}=\frac{\mu_{0} K_{3}^{2} \beta^{\prime \prime}}{(1+\chi) \alpha^{\prime \prime} \rho_{0} \mathrm{~g}}, \mathrm{M}_{3}=\frac{\left(1+\frac{M_{0}}{H_{0}}\right)}{(1+\chi)}, \mathrm{M}_{4}=\frac{\mu_{0} K_{1} K_{2} \beta^{\prime}}{(1+\chi) \alpha \rho_{0} \mathrm{~g}}, \mathrm{M}_{4}^{\prime}=\frac{\mu_{0} K_{2} K_{3} \beta^{\prime \prime}}{(1+\chi) \alpha^{\prime} \rho_{0} \mathrm{~g}}, \\
& \mathrm{M}_{4}^{\prime \prime}=\frac{\mu_{0} K_{1} K_{3} \beta}{(1+\chi) \alpha^{\prime \prime} \rho_{0} \mathrm{~g}}, \mathrm{M}_{5}=\frac{M_{4}}{M_{1}}=\frac{M_{1}^{\prime}}{M_{4}^{\prime}}=\frac{K_{2} \beta^{\prime}}{K_{1} \beta}=\frac{M_{1}^{\prime \prime}}{M_{4}^{\prime \prime}}=\frac{K_{3} \beta^{\prime \prime}}{K_{1} \beta}, M_{\delta}=\frac{K_{1} \beta \mathrm{d}}{\mathrm{H}_{0}(1+\chi) \sqrt{R}}, M_{\delta}{ }^{\prime}=\frac{K_{2} \beta^{\prime} \mathrm{d}}{\mathrm{H}_{0}(1+\chi) \sqrt{S_{1}}} \\
& M_{\delta}^{\prime \prime}=\frac{K_{3} \beta^{\prime \prime} \mathrm{d}}{\mathrm{H}_{0}(1+\chi) \sqrt{S_{2}}}, L_{e}=\frac{K}{K^{\prime}}=\frac{K}{K^{\prime \prime}},
\end{aligned}
$$

Where, $R$ is the Rayleigh number, $S_{1} \& S_{2}$ are the solute Rayleigh number, $M_{1}{ }^{\prime} \& M_{1}^{\prime \prime}$ are the effect of magnetization due to salinity, $\mathrm{M}_{5}$ represent the ratio of the salinity effect on magnetic field to pyromagnetic coefficient and $L_{e}$ is the Lewis number.

The functions $\mathbf{q}, \theta, \gamma, \gamma^{\prime}, \phi_{1}, \phi_{2}, \phi_{3}$ must subject to the boundary conditions and we suppose that $\mathbf{q}, \theta, \gamma, \gamma^{\prime}$, $\phi_{1}, \phi_{2}, \phi_{3}$ are periodic in $\mathrm{x}, \mathrm{y}$ with periods $\frac{2 \pi}{a_{i}}$ for $\mathrm{i}=1,2$ respectively and the surfaces are stress free so that

$\mathrm{w}=0, \mathrm{u}_{\mathrm{z}}=0, \mathrm{v}_{\mathrm{z}}=0, \theta=0, \gamma=0, \gamma^{\prime}=0, \phi_{1 \mathrm{z}}=0, \phi_{2 \mathrm{z}}=0, \phi_{3 \mathrm{z}}=0$ at $\mathrm{z}= \pm \frac{1}{2}$

In order to exclude the rigid motions, we assume that the mean values of $u$ and $v$ are zero (Wells and Kloeden [31]) i.e. we require

$$
\int_{v} u d V=\int_{v} v d V=0
$$

where $\mathrm{V}=\left[0, \frac{2 \pi}{a_{1}}\right] \times\left[0, \frac{2 \pi}{a_{2}}\right] \times\left[\frac{-1}{2}, \frac{1}{2}\right]$ is the typical periodicity cell.

\section{Nonlinear analysis}

To study the nonlinear stability of triple diffusive convection, we derive an energy equation of the form

$$
\frac{d E}{d t}=\mathrm{I}_{0}-\mathrm{D}_{0}+\mathrm{N}_{0}
$$

where 
$\mathrm{E}=\frac{1}{2}\|\theta\|^{2}+\frac{\lambda_{1}}{2}\|\boldsymbol{q}\|^{2}-\frac{\lambda_{3}}{2}\|\gamma\|^{2}-\frac{\lambda_{5}}{2}\left\|\gamma^{\prime}\right\|^{2}$

with coupling parameters $\lambda_{i}$ and \|\| denote the norm on $\mathrm{L}^{2}(\mathrm{~V})$. The terms $\mathrm{I}_{0}, \mathrm{D}_{0}, \mathrm{~N}_{0}$ are as follow:

$$
\begin{aligned}
& \mathrm{I}_{0}=\sqrt{R}\left\{1+\lambda_{1}\left(1+\mathrm{M}_{1}-\mathrm{M}_{4}\right)\right\}\langle w \theta\rangle-\sqrt{S_{1}}\left\{\lambda_{3}+\frac{\lambda_{1}}{L_{e}}\left(1+\mathrm{M}_{4}{ }^{\prime}-\mathrm{M}_{1}{ }^{\prime}\right)\right\}\langle\mathrm{w} \gamma\rangle-\sqrt{S_{2}}\left(\lambda_{5}+\frac{\lambda_{1}}{L_{e}}\left(\mathrm{M}_{4}{ }^{\prime \prime}-\mathrm{M}_{1}{ }^{\prime \prime}\right)\right\}\langle\mathrm{w} \gamma\rangle \\
& -\sqrt{R} \lambda_{1}\left(\mathrm{M}_{1}-\mathrm{M}_{4}\right)\left\langle\mathrm{w} \phi_{1 \mathrm{Z}}\right\rangle+\frac{\sqrt{S_{1}}}{L_{e}} \lambda_{1}\left(\mathrm{M}_{4}^{\prime}-\mathrm{M}_{1}^{\prime}\right)\left\langle\mathrm{w} \phi_{2 \mathrm{Z}}\right\rangle-\frac{\sqrt{S_{2}}}{L_{e}} \lambda_{1}\left(\mathrm{M}_{4}^{\prime \prime}-\mathrm{M}_{1}^{\prime \prime}\right)\left\langle\mathrm{w} \phi_{3 \mathrm{Z}}\right\rangle-\lambda_{2}\left\langle\phi_{1} \theta_{\mathrm{Z}}\right\rangle+\lambda_{4} \\
& \left\langle\phi_{2} \gamma_{\mathrm{Z}}\right\rangle+\lambda_{6}\left\langle\phi_{3} \gamma_{\mathrm{Z}}\right\rangle \\
& \mathrm{D}_{0}=\|\nabla \theta\|^{2}+\lambda_{1}\left(1+\delta \mathrm{M}_{3}\right)\|\nabla q\|^{2}-\frac{\lambda_{3}}{L_{e}}\|\nabla \gamma\|^{2}-\frac{\lambda_{5}}{L_{e}}\left\|\nabla \gamma^{\prime}\right\|^{2}+\lambda_{2} \mathrm{M}_{3}\left\|\nabla \phi_{1}\right\|^{2}-\lambda_{2}\left(\mathrm{M}_{3}-1\right)\left\|\nabla \phi_{1 z}\right\|^{2} \\
& -\lambda_{4} \mathrm{M}_{3}\left\|\nabla \phi_{2}\right\|^{2}\left(\mathrm{M}_{3}-1\right)\left\|\nabla \phi_{2 z}\right\|^{2}-\lambda_{6} \mathrm{M}_{3}\left\|\nabla \phi_{3}\right\|^{2}-\lambda_{6}\left(\mathrm{M}_{3}-1\right)\left\|\nabla \phi_{3 z}\right\|^{2} \\
& \mathrm{~N}_{0}=\lambda_{1} \mathrm{M}_{1}\left\langle\boldsymbol{q} \nabla \theta \phi_{1 z}\right\rangle-\frac{\lambda_{1} \sqrt{M_{4}} \sqrt{M_{4}^{\prime}}}{\sqrt{L_{e}}}\left\langle\mathbf{q} \nabla \theta \phi_{2 z}\right\rangle-\frac{\lambda_{1} \sqrt{M_{4}} \sqrt{M_{4^{\prime \prime}}}}{\sqrt{L_{e}}}\left\langle\mathbf{q} \nabla \theta \phi_{3 z}\right\rangle+\frac{\lambda_{1} M_{1}^{\prime}}{L_{e}}\left\langle\mathbf{q} \nabla \gamma \phi_{2 z}\right\rangle+\frac{\sqrt{M^{\prime}} \sqrt{M_{4^{\prime \prime}}}}{\sqrt{L_{e}}} \\
& \left\langle\mathbf{q} \nabla \gamma \phi_{1 z}\right\rangle+\frac{\lambda_{1} M_{1}^{\prime \prime}}{L_{e}}\left\langle\mathbf{q} \nabla \gamma^{\prime} \phi_{3 z}\right\rangle+\lambda_{1}\left(M_{3}-\frac{1}{1+\chi}\right)\left[M_{1}\left\langle\phi_{1 x} \boldsymbol{q} \nabla \phi_{1 x}\right\rangle-\frac{\sqrt{M_{4}} \sqrt{M_{4}^{\prime}}}{\sqrt{L_{e}}}\left\{\left\langle\phi_{1 \times} \mathbf{q} \nabla \phi_{2 \times}\right\rangle+\left\langle\phi_{2 x} \boldsymbol{q} \nabla \phi_{1 x}\right\rangle\right\}\right. \\
& -\frac{\sqrt{M_{4}} \sqrt{M_{4}^{\prime \prime}}}{\sqrt{L_{e}}}\left\{\left\langle\phi_{1 \times} \boldsymbol{q} \nabla \phi_{3 \times}\right\rangle+\left\langle\phi_{3 \mathrm{x}} \boldsymbol{q} \nabla \phi_{1 \times}\right\rangle\right\}-\frac{\sqrt{M^{\prime}} \sqrt{M_{4}^{\prime \prime}}}{\sqrt{L_{e}}}\left\{\left\langle\phi_{2 \times} \boldsymbol{q} \nabla \phi_{3 \mathrm{x}}\right\rangle+\left\langle\phi_{3 \mathrm{x}} \boldsymbol{q} \nabla \phi_{2 \mathrm{x}}\right\rangle\right\}+\frac{M_{1}^{\prime}}{L_{e}}\left\langle\phi_{2 \times} \boldsymbol{q} \nabla \phi_{2 \times}\right\rangle \\
& \left.+\frac{M_{1}^{\prime \prime}}{L_{e}}\left\langle\phi_{3 \mathrm{x}} \boldsymbol{q} \nabla \phi_{3 \mathrm{x}}\right\rangle\right]-\lambda_{1}\left(\mathrm{M}_{3}-\frac{1}{1+\chi}\right)\left[\mathrm{M}_{1}\left\langle\phi_{1 \mathrm{y}} \boldsymbol{q} \nabla \phi_{1 \mathrm{y}}\right\rangle-\frac{\sqrt{M_{4}} \sqrt{M_{4}^{\prime}}}{\sqrt{L_{e}}}\left\{\left\langle\phi_{1 \mathrm{y}} \boldsymbol{q} \nabla \phi_{2 y}\right\rangle+\left\langle\phi_{2 y} \boldsymbol{q} \nabla \phi_{1 y}\right\rangle\right\}\right. \\
& -\frac{\sqrt{M_{4}} \sqrt{M_{4}^{\prime \prime}}}{\sqrt{L_{e}}}\left\{\left\langle\phi_{1 y} \boldsymbol{q} \nabla \phi_{3 y}\right\rangle+\left\langle\phi_{3 y} \boldsymbol{q} \nabla \phi_{1 y}\right\rangle\right\}-\frac{\sqrt{M^{\prime}} \sqrt{M_{4}^{\prime \prime}}}{\sqrt{L_{e}}}\left\{\left\langle\phi_{2 y} \boldsymbol{q} \nabla \phi_{3 y}\right\rangle+\left\langle\phi_{3 y} \boldsymbol{q} \nabla \phi_{2 y}\right\rangle\right\} \\
& \left.+\frac{M_{1}^{\prime}}{L_{e}}\left\langle\phi_{2 y} \boldsymbol{q} \nabla \phi_{2 y}\right\rangle+\frac{M_{1 "}^{\prime \prime}}{L_{e}}\left\langle\phi_{3 y} \boldsymbol{q} \nabla \phi_{3 y}\right\rangle\right]+\lambda_{1}\left(\frac{\chi}{1+\chi}\right)\left[M_{1}\left\langle\phi_{1 z} \boldsymbol{q} \nabla \phi_{1 z}\right\rangle-\frac{\sqrt{M_{4}} \sqrt{M_{4}^{\prime}}}{\sqrt{L_{e}}}\left\{\left\langle\phi_{1 z} \boldsymbol{q} \nabla \phi_{2 z}\right\rangle+\left\langle\phi_{2 z} \boldsymbol{q} \nabla \phi_{1 z}\right\rangle\right\}\right. \\
& -\frac{\sqrt{M_{4}} \sqrt{M_{4}^{\prime \prime}}}{\sqrt{L_{e}}}\left\{\left\langle\phi_{1 z} \boldsymbol{q} \nabla \phi_{3 z}\right\rangle+\left\langle\phi_{3 z} \boldsymbol{q} \nabla \phi_{1 z}\right\rangle\right\}-\frac{\sqrt{M^{\prime}} \sqrt{M_{4}^{\prime \prime}}}{\sqrt{L_{e}}}\left\{\left\langle\phi_{2 z} \boldsymbol{q} \nabla \phi_{3 z}\right\rangle+\left\langle\phi_{3 z} \boldsymbol{q} \nabla \phi_{2 z}\right\rangle\right\}+\frac{M_{1}^{\prime}}{L_{e}}\left\langle\phi_{2 z} \boldsymbol{q} \nabla \phi_{2 z}\right\rangle \\
& \left.+\frac{M_{1}{ }^{\prime \prime}}{L_{e}}\left\langle\phi_{3 z} \boldsymbol{q} \nabla \phi_{3 z}\right\rangle\right]+\lambda_{1} \delta M_{3}\left\{M_{\delta}\left\langle\phi_{1 \times} \mathbf{q} \nabla^{2} \mathbf{q}\right\rangle-M^{\prime}{ }_{\delta}\left\langle\phi_{2 \times} \mathbf{q} \nabla^{2} \mathbf{q}\right\rangle-M_{\delta}{ }^{\prime \prime}\left\langle\phi_{3 \times} \mathbf{q} \nabla^{2} \mathbf{q}\right\rangle-M_{\delta}\left\langle\phi_{1 \mathrm{y}} \mathbf{q} \nabla^{2} \mathbf{q}\right\rangle-M_{\delta}{ }^{\prime}\left\langle\phi_{2 y} \mathbf{q} \nabla^{2} \mathbf{q}\right\rangle\right. \\
& \left.-M_{\delta}{ }^{\prime \prime}\left\langle\phi_{3 \mathbf{y}} \mathbf{q} \nabla^{2} \mathbf{q}\right\rangle\right\}+\lambda_{1} \delta\left\{\left\langle\phi_{1 z} \mathbf{q} \nabla^{2} \mathbf{q}\right\rangle-M^{\prime}{ }_{\delta}\left\langle\phi_{2 z} \mathbf{q} \nabla^{2} \mathbf{q}\right\rangle-M_{\delta}{ }^{\prime \prime}\left\langle\phi_{3 z} \mathbf{q} \nabla^{2} \mathbf{q}\right\rangle\right\}-\lambda_{1} \delta M_{\delta}\left\langle\theta \mathbf{q} \nabla^{2} \mathbf{q}\right\rangle+\lambda_{1} \delta M_{\delta}{ }^{\prime}\left\langle\gamma \mathbf{q} \nabla^{2} \mathbf{q}\right\rangle \\
& +\lambda_{1} \delta M_{\delta}^{\prime \prime}\left\langle\gamma^{\prime} \boldsymbol{q} \nabla^{2} \mathbf{q}\right\rangle
\end{aligned}
$$

where $\lambda_{1}, \lambda_{2}, \lambda_{3}, \lambda_{4}, \lambda_{5}, \lambda_{6}$ are positive coupling parameters and \langle\rangle is the inner product on $\mathrm{L}^{2}(\mathrm{~V})$.

In equation (24), it is seen that the energy of the system is consumed due to the solute concentrations (i.e. -ve sign with $\left.\frac{\lambda_{3}}{2}\|\gamma\|^{2} \&-\frac{\lambda_{5}}{2}\left\|\gamma^{\prime}\right\|^{2}\right)$. Now, it can be assumed that the energy is consumed, due to solute concentrations is less than the energy produced due to velocity and temperature. Also the energy dissipated by the solute concentrations is less than the energy dissipated by the velocity, temperature and magnetization. These assumptions will ensure that all the terms on the right-hand side of the equations (24) \& (26) are always less than the left - hand side of that equation.

Thus from equation (23), we have

$\frac{d E}{d t} \leq-\mathrm{a}_{0} \mathrm{D}_{0}+\mathrm{N}_{0}$

with $\mathrm{a}_{0}=1-\mathrm{m}(>0)$ where

$\mathrm{m}=\max _{H} \frac{I_{0}}{D_{0}}$

and $\mathrm{H}$ is the space of admissible solution. 
Triple- Diffusive Convection In A Magnetized Ferrofluid With Mfd Viscosity: A Nonlinear Stability

In order to dominate the nonlinear terms and for studying the nonlinear stability, we now introduce the generalized energy functional as

$\mathrm{V}_{\mathrm{g}}(\mathrm{t})=\mathrm{E}(\mathrm{t})+\mathrm{b}_{0} \mathrm{E}_{1}(\mathrm{t})$

where $b_{0}$ is a positive coupling parameter to be chosen and the complementary energy $E_{1}(t)$ is given by

$\mathrm{E}_{1}(\mathrm{t})=\frac{1}{2}\|\nabla \theta\|^{2}+\frac{1}{2}\|\nabla q\|^{2}+\frac{1}{2}\|\nabla \gamma\|^{2}+-\frac{1}{2}\left\|\nabla \gamma^{\prime}\right\|^{2}+\frac{1}{2}\left\|\nabla^{2} \theta\right\|^{2}+\frac{1}{2}\left\|\nabla^{2} \gamma\right\|^{2}+\frac{1}{2}\left\|\nabla^{2} \gamma^{\prime}\right\|^{2}$

III. The eigenvalue problem of nonlinear analysis

Now we use the calculus of variation to find the maximum problem at the critical argument $m_{1}=1$ in equation (29). The associated Euler-Lagrange equations, after taking the transformations

$\widehat{\boldsymbol{q}}=\sqrt{\lambda_{1}} \mathbf{q}, \hat{\phi}_{1=\sqrt{\lambda_{2}}} \phi_{1}, \hat{\gamma}=\sqrt{\lambda_{3}} \gamma, \widehat{\phi}_{2}=\sqrt{\lambda_{4}} \phi_{4}, \hat{\gamma}^{\prime}=\sqrt{\lambda_{5}} \gamma^{\prime}, \hat{\phi}_{3}=\sqrt{\lambda_{6}} \phi_{3}$,

and dropping the caps are

$$
\begin{aligned}
& 2\left(1+\delta \mathrm{M}_{3}\right) \nabla^{2} \mathbf{q}+\frac{\sqrt{R}}{\sqrt{\lambda_{1}}}\left\{1+\lambda_{1}\left(1+\mathrm{M}_{1}-\mathrm{M}_{4}\right)\right\} \theta \widehat{\boldsymbol{k}}-\frac{\sqrt{\boldsymbol{s}_{\mathbf{1}}}}{\sqrt{\lambda_{1}} \sqrt{\lambda_{3}}}\left\{\lambda_{3}+\frac{\lambda_{1}}{L_{e}}\left(1+\mathrm{M}_{4}{ }^{\prime}-\mathrm{M}_{1}{ }^{\prime}\right)\right\} \gamma \widehat{\boldsymbol{k}}-\frac{\sqrt{S_{2}}}{\sqrt{\lambda_{1}} \sqrt{\lambda_{5}}}\left(\lambda_{5}+\frac{\lambda_{1}}{L_{e}}\right. \\
& \left.\left(\mathrm{M}_{4}^{\prime \prime}-\mathrm{M}_{1}^{\prime \prime}\right)\right\} \gamma^{\prime} \widehat{\boldsymbol{k}}-\frac{\sqrt{\lambda_{1}}}{\sqrt{\lambda_{4}}} \sqrt{R}\left(\mathrm{M}_{1}-\mathrm{M}_{4}\right) \phi_{1 z} \widehat{\boldsymbol{k}}+\frac{\sqrt{\lambda_{1}}}{\sqrt{\lambda_{4}}} \frac{\sqrt{S_{1}}}{L_{e}}\left(\mathrm{M}_{4}^{\prime}-\mathrm{M}_{1}^{\prime}\right) \phi_{2 z} \widehat{\boldsymbol{k}}+\frac{\sqrt{\lambda_{1}}}{\sqrt{\lambda_{6}}} \frac{\sqrt{S_{2}}}{L_{e}}\left(\mathrm{M}_{4}^{\prime \prime}-\mathrm{M}_{1}^{\prime \prime}\right) \\
& \phi_{32} \widehat{\boldsymbol{k}}-2 \nabla \mathrm{p}=0 \\
& 2 \nabla^{2} \theta+\sqrt{R}\left\{1+\lambda_{1}\left(1+\mathrm{M}_{1}-\mathrm{M}_{4}\right)\right\} \frac{1}{\sqrt{\lambda_{1}}} w+\sqrt{\lambda_{2}} \phi_{1 z}=0 \\
& \frac{2}{L_{e}} \nabla^{2} \gamma+\sqrt{S_{1}}\left\{\lambda_{3}+\frac{\lambda_{1}}{L_{e}}\left(1+\mathrm{M}_{4}^{\prime}-\mathrm{M}_{1}^{\prime}\right)\right\} \frac{1}{\sqrt{\lambda_{1}} \sqrt{\lambda_{3}}} \mathrm{w}+\frac{\sqrt{\lambda_{4}}}{\sqrt{\lambda_{3}}} \phi_{2 Z}=0 \\
& \frac{2}{L_{e}} \nabla^{2} \gamma^{\prime}+\sqrt{S_{2}}\left(\lambda_{5}+\frac{\lambda_{1}}{L_{e}}\left(\mathrm{M}_{4}^{\prime \prime}-\mathrm{M}_{1}^{\prime \prime}\right)\right\} \frac{1}{\sqrt{\lambda_{1}} \sqrt{\lambda_{5}}} \mathrm{w}+\frac{\sqrt{\lambda_{6}}}{\sqrt{\lambda_{5}}} \phi_{3 \mathrm{Z}}=0 \\
& 2 \mathrm{M}_{3} \nabla^{2} \phi_{1}-2\left(\mathrm{M}_{3}-1\right) \phi_{1 z z}+\sqrt{R} \frac{\sqrt{\lambda_{1}}}{\sqrt{\lambda_{2}}}\left(\mathrm{M}_{1}-\mathrm{M}_{4}\right) \mathrm{w}_{2}-\sqrt{\lambda_{2}} \theta_{2}=0 \\
& 2 M_{3} \nabla^{2} \phi_{2}-2\left(M_{3}-1\right) \phi_{2 z z}+\frac{\sqrt{\lambda_{1}}}{\sqrt{\lambda_{4}}} \frac{\sqrt{S_{1}}}{L_{e}}\left(M_{1}^{\prime}-M_{4}^{\prime}\right) w_{z}-\sqrt{\lambda_{2}} \gamma_{z}=0 \\
& 2 M_{3} \nabla^{2} \phi_{3}-2\left(M_{3}-1\right) \phi_{3 z z}+\frac{\sqrt{\lambda_{1}}}{\sqrt{\lambda_{6}}} \frac{\sqrt{S_{2}}}{L_{e}}\left(M_{1}^{\prime \prime}-M_{4}^{\prime \prime}\right) w_{2}-\sqrt{\lambda_{2}} \gamma^{\prime}{ }_{2}=0
\end{aligned}
$$

After taking the third component of the curl curl of equation (33), we have

$$
\begin{aligned}
& 2\left(1+\delta \mathrm{M}_{3}\right) \nabla^{4} \mathrm{w}+\frac{\sqrt{R}}{\sqrt{\lambda_{1}}}\left\{1+\lambda_{1}\left(1+\mathrm{M}_{1}-\mathrm{M}_{4}\right)\right\} \nabla^{2}{ }_{1} \theta-\frac{\sqrt{S_{1}}}{\sqrt{\lambda_{1}} \sqrt{\lambda_{3}}}\left\{\lambda_{3}+\frac{\lambda_{1}}{L_{e}}\left(1+\mathrm{M}_{4}{ }^{\prime}-\mathrm{M}_{1}{ }^{\prime}\right)\right\} \nabla^{2}{ }_{1} \gamma \\
& -\frac{\sqrt{S_{2}}}{\sqrt{\lambda_{1}} \sqrt{\lambda_{5}}}\left(\lambda_{5}+\frac{\lambda_{1}}{L_{e}}\left(\mathrm{M}_{4}{ }^{\prime \prime}-\mathrm{M}_{1}{ }^{\prime \prime}\right)\right\} \nabla^{2}{ }_{1} \gamma^{\prime}-\frac{\sqrt{\lambda_{1}}}{\sqrt{\lambda_{4}}} \sqrt{R}\left(\mathrm{M}_{1}-\mathrm{M}_{4}\right) \nabla^{2}{ }_{1} \phi_{1 \mathrm{Z}}+\frac{\sqrt{\lambda_{1}}}{\sqrt{\lambda_{4}}} \frac{\sqrt{S_{1}}}{L_{e}}\left(\mathrm{M}_{4}{ }^{\prime}-\mathrm{M}_{1}{ }^{\prime}\right) \nabla^{2}{ }_{1} \phi_{2 Z}+\frac{\sqrt{\lambda_{1}}}{\sqrt{\lambda_{6}}} \frac{\sqrt{S_{2}}}{L_{e}} \\
& \left(\mathrm{M}_{4}{ }^{\prime \prime}-\mathrm{M}_{1}{ }^{\prime \prime}\right) \nabla^{2}{ }_{1} \phi_{3 \mathrm{Z}}=0
\end{aligned}
$$

Now, we assume a plane tilting form

$\left(\mathrm{w}, \theta, \gamma, \gamma^{\prime}, \phi_{1}, \phi_{2}, \phi_{3}\right)=\left[\mathrm{W}(\mathrm{z}), \Theta(\mathrm{z}), \Gamma(\mathrm{z}), \Psi(\mathrm{z}), \phi_{1}(\mathrm{z}), \phi_{2}(\mathrm{z}), \phi_{3}(\mathrm{z})\right] \mathrm{g}(\mathrm{x}, \mathrm{y})$

Where $\nabla^{2}{ }_{1} g+\mathrm{a}^{2} \mathrm{~g}=0$, 'a' being the wave number (Chandrasekhar [1981])

The boundary conditions at the free-free surface are

$\mathrm{W}=\mathrm{D}^{2} \mathrm{~W}=\Theta=\Gamma=\Psi=\mathrm{D} \phi_{1}=\mathrm{D} \phi_{2}=\mathrm{D} \phi_{3}=0$ at $\mathrm{z}= \pm \frac{1}{2}$,

The exact solution subject to these boundary conditions is written in the form 
$\mathrm{W}=\mathrm{A}_{1} \cos \pi z, \Theta=\mathrm{A}_{2} \cos \pi z, \mathrm{D} \phi_{3}=\mathrm{A}_{3} \cos \pi z$,

$\phi_{1}=\left(\frac{A_{3}}{\pi}\right) \sin \pi z, \Gamma=\mathrm{A}_{4} \cos \pi z, \mathrm{D} \phi_{2}=\mathrm{A}_{5} \cos \pi z$,

$\phi_{2}=\left(\frac{A_{5}}{\pi}\right) \sin \pi z, \Psi=\mathrm{A}_{6} \cos \pi z, \mathrm{D} \phi_{3}=\mathrm{A}_{7} \cos \pi z$,

$\phi_{3}=\left(\frac{A_{7}}{\pi}\right) \sin \pi z$,

Where $A_{1}, A_{2}, A_{3}, A_{4}, A_{5}, A_{6}, A_{7}$ are constants. Using the plane tiling form and substituting solution (44), we get equations involving coefficients of $A_{1}, A_{2}, A_{3}, A_{4}, A_{5}, A_{6}, A_{7}$. For the existence of non-trivial solutions, the determinant of the coefficients of $A_{1}, A_{2}, A_{3}, A_{4}, A_{5}, A_{6}, A_{7}$ must vanish. This determinant on simplification yields the energy thermal Rayleigh number $\mathrm{R}_{\mathrm{e}}^{\prime}$ and then we can performs the optimization

$\mathrm{R}_{\mathrm{e}}=\max _{\lambda_{1}, \lambda^{\prime} 2, \lambda_{3}, \lambda^{\prime} \lambda_{4}, \lambda_{5}, \lambda_{6}^{\prime}}, \min _{x} \mathrm{R}_{\mathrm{e}}^{\prime}\left(\lambda_{1}, \lambda_{2}^{\prime}, \lambda_{3}, \lambda^{\prime}{ }_{4}, \lambda_{5}, \lambda_{6}^{\prime}, M_{1}, M_{3}, M_{5}, M_{1}^{\prime}, M^{\prime \prime}{ }_{1}, \delta, L_{e}, \mathrm{~S}_{1}, \mathrm{~S}_{2}\right)$

where $\mathrm{R}_{\mathrm{e}}^{\prime}=\frac{R_{e}}{\pi^{4}}, \mathrm{~S}_{1}=\frac{S}{\pi^{4}} \quad \mathrm{x}=\frac{a^{2}}{\pi^{2}}, \lambda^{\prime}{ }_{2}=\frac{\lambda_{2}}{\pi^{2}}, \lambda^{\prime}{ }_{4}=\frac{\lambda_{4}}{\pi^{2}}, \lambda_{6}^{\prime}=\frac{\lambda_{6}}{\pi^{2}}$

To achieve this we need careful selection of $\lambda_{1}, \lambda_{2}^{\prime}, \lambda_{3}, \lambda_{4}^{\prime}, \lambda_{5}, \lambda_{6}^{\prime}$ and are found to be

$$
\begin{aligned}
& \lambda_{1}=\frac{1}{1+M_{1}\left(1-M_{5}\right)}, \quad \lambda_{2}^{\prime}=\frac{(1+x) M_{1}\left(1-M_{5}\right)}{1+M_{1}\left(1-M_{5}\right)}, \quad \lambda_{3}=\frac{1+M_{1}^{\prime}\left(\frac{1}{M_{5}}-1\right)}{L_{e}\left[1+M_{1}\left(1-M_{5}\right)\right]}, \\
& \lambda_{4}^{\prime}=\frac{(1+x) M^{\prime}{ }_{1}\left(\frac{1}{M_{5}}-1\right)}{L_{e}^{2}\left[1+M_{1}\left(1-M_{5}\right)\right]}, \lambda_{5}=\frac{1+M^{\prime \prime}{ }_{1}\left(\frac{1}{M_{5}}-1\right)}{L_{e}\left[1+M_{1}\left(1-M_{5}\right)\right]}, \lambda_{6}^{\prime}=\frac{(1+x) M^{\prime \prime}{ }_{1}\left(\frac{1}{M_{5}}-1\right)}{L_{e}{ }^{2}\left[1+M_{1}\left(1-M_{5}\right)\right]}
\end{aligned}
$$

Using Equation (46) in (45), we have

$$
\mathrm{R}_{\mathrm{e}}=\frac{\left\{4\left(1+x M_{3}\right)-M_{1}\left(1-M_{5}\right) /\left[1+M_{1}\left(1-M_{5}\right)\right]\right\}\left[\left\{(1+x)^{3}\left(1+\delta M_{3}\right)\right\}+\mathrm{x} S_{1}\left\{1+M_{1}{ }^{\prime}\left(\frac{1}{M_{5}}-1\right)\right\}+\mathrm{x} S_{2}\left\{1+M_{1}{ }^{\prime \prime}\left(\frac{1}{M_{5}}-1\right)\right\}\right]}{\mathrm{x}\left\{4\left(1+\mathrm{x} M_{3}\right)\left[1+M_{1}\left(1-M_{5}\right)\right]-2 M_{1}\left(1-M_{5}\right)\right\}}
$$

For $M_{1}$ sufficiently large, we obtain the magnetic thermal Rayleigh number

$$
N_{e}=M_{1} R_{e}=\frac{\left(3+4 x M_{3}\right)\left[(1+x)^{3}\left(1+\delta M_{3}\right)+x S_{1}\left\{1+M_{1}\left(\frac{1}{M_{5}}-1\right)\right\}+x S_{2}\left\{1+M_{1}{ }^{\prime \prime}\left(\frac{1}{M_{5}}-1\right)\right\}\right]}{\mathrm{x}\left\{\left(2+4 \times M_{3}\right)\left(1-M_{5}\right)\right\}}
$$

as a function of $\mathrm{x}, N_{e}$ given by equation (48) attains its minimum, when

$$
P_{5} x^{5}+P_{4} x^{4}+P_{3} x^{3}+P_{2} x^{2}+P_{1} x+P_{0}=0
$$

the coefficients $P_{0}, P_{1}$ $P_{5}$ being quite lengthy, and have not written here. The Newton-Raphson method is used to determine the values of critical wave number in nonlinear stability results by the condition

$$
\frac{d N_{e}}{d x}=0
$$

With $\mathrm{x}$ determined as a solution of Equation (49), Equation (48) will give the required critical magnetic thermal Rayleigh number $N_{c e}$. In the absence of solute and MFD viscosity, Equation (48) reduces to

$N_{e}=\frac{\left(3+4 x M_{3}\right)(1+x)^{3}}{x\left(2+4 x M_{3}\right)}$

For analyzing the linear instability results, we use the non-dimensional Equations (13)-(20), neglecting the nonlinear terms. We again perform the standard stationary mode analysis and look for the solution of these equations in the form of Equation (42). The boundary conditions in the present case are same i.e. Equation (43). After following the same procedure as stated earlier in the energy stability case, we have

$R_{\ell}=\frac{(1+x)^{3}\left(1+\delta M_{3}\right)\left(1+x M_{3}\right)+x S_{1}\left[1+x M_{3}+x M_{1}^{\prime} M_{3}\left(\frac{1}{M_{5}}-1\right)+x S_{2}\left[1+x M_{3}+x M_{1}^{\prime \prime} M_{3}\left(\frac{1}{M_{5}}-1\right)\right]\right.}{x\left[1+x M_{3}+x M_{1} M_{3}\left(1-M_{5}\right)\right]}$ 
We again consider the magnetic thermal Rayleigh number $N_{e}$ depends on the parameter $M_{3}$. For $M_{1}$ sufficiently large, the linear critical magnetic thermal Rayleigh number is

$N_{\ell}=\frac{(1+x)^{3}\left(1+\delta M_{3}\right)\left(1+x M_{3}\right)+x S_{1}\left[1+x M_{3}+x M_{1}^{\prime} M_{3}\left(\frac{1}{M_{5}}-1\right)+x S_{2}\left[1+x M_{3}+x M_{1}^{\prime \prime} M_{3}\left(\frac{1}{M_{5}}-1\right)\right]\right.}{x^{3} M_{3}\left(1-M_{5}\right)}$

In the absence of the solute and MFD viscosity, Equation (53) reduces to

$N_{\ell}=\frac{(1+x)^{3}\left(1+x M_{3}\right)}{x^{2} M_{3}}$

which is in good agreement with the previous published (Finlayson[1970] )

There are instances in which the two theories coincide. This is true for the classical Bénard problem. In the absence of magnetic parameters $\left(M_{1}=0, M_{1}^{\prime}=0, M^{\prime \prime}{ }_{1}=0\right.$, and $\left.M_{3}=0\right)$, we obtain

$R_{\ell}=\frac{(1+x)^{3}}{x}+S_{1}+S_{2}=\mathrm{R}_{\mathrm{e}}$

In the absence of solutes (i.e. $S_{1}=0 \& S_{2}=0$ ), this further simplifies to

$R_{\ell}=\frac{(1+x)^{3}}{x}=\mathrm{R}_{\mathrm{e}}$

Thus in both the cases the linear instability boundary is equal to linear stability boundary. Here, the energy method leads to the result that arbitrary subcritical instabilities are not possible, which is in good agreement with the previous published work (Joseph[1965'1966]). Thus, for lower values of magnetic parameters, this coincidence is immediately lost.

\section{Results and discussion}

The critical magnetic thermal Rayleigh numbers $N_{c e}, N_{c \ell}$ depend upon $M_{3}, M_{1}^{\prime}, M^{\prime \prime}{ }_{1}, S_{1}, S_{2}$ and $M_{5}$. It has been seen that in the absence of MFD viscosity $(\delta)$, both $N_{c e}, N_{c \ell}$ decrease as $M_{3}$ increases, there by showing the destabilizing effect of $M_{3}$. It has also been observed that in the presence of MFD viscosity $(\delta)$, both $N_{c e}, N_{c \ell}$ decrease for lower values of $M_{3}$ and increase for higher values of $M_{3}$. Thus the MFD viscosity increases with the increase of $N_{c e}, N_{c l}$, hence showing the stabilizing effect of MFD viscosity. In the absence of MFD viscosity, the variation in magnetization releases extra energy, which adds up to the thermal energy to destabilize the system. So, in the absence of the MFD viscosity, magnetization always has a destabilizing effect. The presence of MFD viscosity gives rise to a resistive force. It (force) has the tendency to slow down the motion of the fluid in the boundary layer, thus inducing the heat transfer from bottom to top. The decrease in heat transfer is responsible for delaying the onset of convection. Thus, the MFD viscosity promotes stabilization. Since variation in magnetization leads to change in viscosity, hence for large values of coefficient of the MFD viscosity $(\delta)$ and large values of magnetization $\left(M_{3}\right)$, the resistive viscous force overcomes the energy released, due to increase in magnetization and thus delays the onset of convection. Hence, increase in magnetization stabilizes the system, and so magnetization plays a dual role depending upon the values of coefficient of the MFD viscosity. Also it is observed that the values of $N_{c \ell}$ are always greater than those of $N_{c e}$ , and this is quite obvious from the fact that linear stability theory gives sufficient conditions for instability, while the energy stability theory gives the sufficient condition for stability. Thus, the difference between the values of $N_{c e}$ and $N_{c \ell}$ reveals that there is a band of Rayleigh numbers where subcritical instability may arise. One can note that this band decreases as $M_{3}$ increases.

The solutes gradients $S_{1} \& S_{2}$ have stabilizing effect, because both $N_{c e}, N_{c \ell}$ increases as solute gradients increases. One can note that the subcritical instability region expands with the increase of solute gradients . Here, in this case heating expands the fluid at the bottom of the layer and this in turn wants to expand, thereby enhance the motion due to thermal convection. On the other side, the heavier salts at the lower part of the layer have exactly the opposite effect and these act to prevent motion through convective overturning. Thus, these two effects are competing against each other. Due to this, the linear theory of instability does not always capture the effect of instability completely and instabilities might arise before the threshold is reached, as we have obtained in this problem. 


\section{Conclusions}

In this paper a nonlinear stability analysis of triple- diffusive convection in a magnetized ferrofluid with magnetic field -dependent viscosity has been investigated. It has been observed that the boundaries of nonlinear stability and linear instability analyses do not intersect. The MFD viscosity and solute gradients always delay the onset of convection. We have derived a nonlinear stability threshold very close to the linear instability one. It has been seen that the magnetic mechanism alone can induce subcritical region of instability. The comparison between the linear and energy stability reveals that for convection problem in ferrofluids, the linear critical magnetic thermal Rayleigh number is higher in values than the nonlinear (energy) critical magnetic thermal Rayleigh number, which shows the possibility of the existence of subcritical instability . It is important to realize that the subcritical instability region decreases as magnetization increases. We also observe that solute gradients cannot induce subcritical region of instability, but in magnetic mechanism, this region expands with the increase of solute gradients. In non-ferrofluids, it is verified that the global stability Rayleigh number is exactly the same as that of linear instability.

\section{References}

[1] A. Abraham, Rayleigh-Be'nard convection in a micropolar ferromagnetic fluid, Int. J. Eng. Sci. 40 (4), 2002,449-460.

[2] A. J. Pearlstein et.al., The onset of convective instability in a triply diffusive fluid layer, Journal of Fluid Mechanics, 202, 1989, $443-465$.

[3] B.A. Finlayson, Convective instability of ferromagnetic fluids, J. Fluid Mech.40 (4), 1970, 753-767.

[4] B. Straughan and D.W. Walker, Multi component diffusion and penetrative convection,Fluid DynamicsResearch, Vol. 19, $1997,77-89$.

[5] B. Straughan, A sharp nonlinear stability threshold in rotating porous convection, Proc. Roy. Soc. London. A. vol. 457, 2001,87-93.

[6] B. Straughan, The energy method, Stability, and Nonlinear Convection, New York, Springer Verlag, 2004

[7] B. Straughan, Global nonlinear stability in porous convection with a thermal non-equilibrium model ,proc. R. soc. A. vol.462, 2006, 409-418.

[8] C .Oldenberg and K. Pruess, Layered thermohaline convection in hypersaline geothermal systems.Transport in Porous Media, vol. $33,1998,29-63$.

[9] D.A.Nield and A. Bejan, Convection in Porous Media, New York, Springer, 1998.

[10] D. D. Joseph, On the stability of the Boussinesq equations, Cambridge University Press, 1965.

[11] D. D. Joseph, Nonlinear stability of the Boussinesq equations by the method of energy, Arch. Ration. Mech. Anal., vol.20, $1966,59-71$

[12] D.P. Lalas and S. Carmi ,Thermoconvective stability of ferrofluids, Phys. Fluids 14 (2), 1971, $436-438$.

[13] F. Mc. W. Orr, Stability or instability of the steady motion of perfect liquid, Proc. Roy. Irish. Acad. Sect. A. vol.37, 1970,69-138

[14] H.C. Brinkman, A calculation of the viscous force exerted by a flowing fluid on a dense swarm of particle, Applied Science Research, A., 1947, I27-34.

[15] Hill. A. Antony and Carr. Magda, Nonlinear stability of the one-domain approach to modelling convection in superposed fluid and porous layers Proc. R. Soc. Vol. 466 No. 2121, 2010,2695-2705.

[16] James. Serrin, Poiseuille and Couette flow of non-Newtonian fluids, Z. angew.Math. Mech., vol. 39, 1959,295-305.

[17] M. I. Shliomis, Magnetic fluids, Sov. Phys. Usp. 17, 1974,153-169.

[18] N. Banu and D.A.S Rees, Onset of Darcy-Bénard convection using a thermal non-equilibrium model. Int. J. Heat Mass Transfer. $45,2002,2221-2228$.

[19] O'Sullivan et.al., State of the of geothermal reserviour simulation. Geothermics, vol.30, 2000, 395-429.

[20] 0. Reynolds, On the dynamical theory of incompressible viscous fluids and determination of criterion, Phil. Trans.R.Soc. A 186, $1895,123-164$

[21] P.J. Stiles and M. Kagan, Thermoconvective instability of a horizontal layer of ferrofluid in a strong vertical magnetic field, J. Magn. Magn. Mater. 85 (1-3) , 1990, 196-198

[22] R.E. Rosensweig, Ferrohydrodynamics, Cambridge University Press, Cambridge, 1985.

[23] R.C. Sharma and P. Kumar, On micropolar fluids heated from below in hydromagnetics in porous medium, Czech. J. Phys. Vol.47, 1997, 637-647.

[24] R. Lopez Amalia et.al., Effect of rigid boundaries on the onset of convective instability in a triply diffusive fluid layer, Phys. Fluids A. vol. 2, 1990,897.

[25] S. Chandrasekhar, Hydrodynamic and Hydromagnetic Stability, Dover Publication, New York, 1981.

[26] S. Venkatasubramanian and P.N. Kaloni , Effects of rotation on the thermoconvective instability of a horizontal layer of Ferrofluids, Int.J. Eng. Sci. vol.32, 1994, 237-256.

[27] Sunil and A. Mahajan, A nonlinear stability analysis for magnetized ferrofluid heated from below, Proc. Roy. Soc. A. vol. 464, 2008, 83-98.

[28] Sunil and A. Mahajan, A nonlinear stability analysis for thermoconvective magnetized ferrofluid saturating a porous medium, Trans. Porous Media. Vol. 76, 2009,327-343.

[29] Suresh Chand, Effect of rotation on triple- diffusive convection in a magnetized ferrofluid with internal angular momentum saturating a porous medium, Applied Mathematical Sciences, Vol. 6, 2012, no.65, 2012,3245-3258.

[30] Suresh Chand, Triple-Diffusive convection in a micropolar ferrofluid in the presence of rotation, IJERT - International Journal of Engineering Research and Technology Vol. 1 Issue 5, July - 2012.

[31] Wells.R. and Kloeden.P (1983), An explicit example of Hopf bifurcation in fluid mechanics, Proc. Roy. Soc.London. Ser. A. vol. 390, 1983, 293-320. 